\title{
Copula-based Analysis of Spatial Distribution Characteristics of Drought in Songnen Grassland
}

\author{
Wurina $^{1}$, Jiquan Zhang ${ }^{{ }^{*}}$, Laiquan ${ }^{1,2}$, Siqin Tong ${ }^{1}$ \\ ${ }^{1}$ College of Environment, Northeast Normal University, Jilin Changchun 130024, China \\ ${ }^{2}$ College of Geographical, Inner Mongolia Normal University, Inner Mongolia Huhhot010022, China
}

\section{基于 Copula 函数的松嫩草原干旱空间分布特征 研究

\author{
乌日娜 ${ }^{1}$, 张继权 ${ }^{1 *}$, 来全 $^{1,2}$, 佟斯琴 ${ }^{1}$ \\ ${ }^{1}$ 东北师范大学环境学院, 吉林长春 130117 \\ 2 内蒙古师范大学地理科学学院, 内蒙古呼和浩特 010022
}

\begin{abstract}
In this paper, daily temperature and daily precipitation data at 15 meteorological stations from 1960 to 2014 in Songnen Grassland were used to analyze the characteristics of spatial distribution of drought. A drought event is defined by the run theory and the drought event is described by two characteristic variables, i.e., drought duration and drought severity. Then, three Archimedean Copula functions are used to construct the joint distribution of two characteristic variables and finally the GIS as spatial statistical analysistool to analyze spatial distribution of the drought return period. The results shows that the Frank Copula well reflect the relationships among drought characteristic variables. The characteristics of the spatial distribution of drought return periods of Songnen grassland are obviously different. The study of return period can provide the scientific basis for risk assessment and drought management for Songnen grassland.
\end{abstract}

Keywords: Drought spatial analysis; Standardized precipitation Evapotranspiration index (SPEI); Copula; Return period

\section{摘要}

本文以松嫩草原 15 个气象站点 1960-2014 年 的日气温和日降水资料为基础, 选用游程理论 识别了草原干旱特征变量, 并利用 3 种 Archimedean Copula 函数分别构建了干旱历 时和干旱强度两个干旱特征变量之间的联合 分布特征, 最后以 GIS 的空间统计分析为工具, 分析了松嫩草原在指定重现期下干旱重现期 和同现期的空间分布特征。研究结果表明: 经 拟合优度评价, Frank Copula 对干旱历时和 干旱强度的拟合度最好; 通过 GIS 空间分析发 现重现期空间分布差异很明显; 各气象站点干 旱程度差异较大。重现期的研究能够为松嫩草 地干旱灾害风险评价与管理工作提供科学依 据。

关键词: 空间分布特征; 标准化降水蒸腾指数 (SPEI); Copula 函数; 重现期

1. 引言

干早由于发生频率高、持续时间长、涉及范围 广、造成的经济损失巨大等特点, 成为所有自

作者简介: 乌日娜 (1988-), 女, 蒙古族, 东北师 范大学博士, 主要从事灾害与风险评价与管理。通 *讯作者简介: 张继权 (1965-), 男, 吉林九台人, 教授, 博士生导师, 主要从事区域灾害与综合风险 评价、预警与应急管理研究。

E-mail:zhangjq022@nenu.edu.cn 


\section{Risk Analysis and Crisis Response in Big Data Era (RAC-16)}

然灾害中破坏最为严重的灾种。其中, 草原干 旱灾害给牧区造成的破坏尤其要引起重视。中 国草原的主体位于在年降水量小于 400 毫米 的北部、西部广大地区, 干旱发生频繁, 易形 成畜牧业灾害。其危害程度, 位于我国牧区各 类畜牧业气象灾害之首。在中国的牧区, 对干 旱灾害的防灾减灾能力较为低下, 农牧民防灾 避灾知识缺乏, 在一定程度上增加了灾害造成 的损失。因此, 进行草原干旱时空分布特征分 析, 实现草原干旱灾害管理已经成为一项十分 紧迫的任务, 对保护草原生态安全、促进牧区 社会经济可持续发展具有重大意义。

干旱形成原因复杂, 受多种因素影响, 因此需 要建立干旱评价指标。目前气象干旱指标主要 有降水距平百分率、标准化降雨量指数 (Standard Precipitation Index, SPI)、帕 默尔指数 (PDSI)、降水 Z 指数 (Moisture Anomaly Index, Z-index)、综合指数 (CI)、 有效干旱指数 (Effective Drought Index, EDI )、Bhalme-Mooley index (BMI)、SPEI (Standard Precipitation Evapotranspire Index）等。每种评价指标都有其优势和不足 之处, 对不同的研究地区应选择合适的干旱评 价指标。而且多数对干旱时空演变规律的研究 都侧重关注干旱历时和干旱强度两个方面。传 统的统计方法计算联合分布时要求二变量的 边缘分布为相同的分布类型, 但是干旱历时和 干旱强度的分布类型并不相同，使得利用传 统的统计方法分析二者的联合分布特征变得 困难。为了解决以上研究的不足, 本文将选取
SPEI 指数作为干旱分析指标。采用游程理论 从干旱指数中分离出干旱灾害事件的干旱历 时和干旱强度, 再利用 Copula 函数建立起两 者的二维概率密度函数, 进而计算不同历时和 不同强度干旱时间的概率。最后通过估计指定 重现期下的各站的干早重现期, 以及运用 GIS 的空间差值功能对其进行反距离权重差值并 利用 ARCGIS 的空间分布功能进行空间展布, 并深入分析草原干旱的空间分布特征, 从而为 松嫩草地干旱灾害风险评价、旱情评估和风险 管理提供有效的科学依据.

\section{2. 研究区概况与数据来源}

本研究以松嫩草原为研究区 (图 1), 研究区 位于东北的中部, 地处北纬 $43^{\circ} 30^{\prime} \sim$ $48^{\circ} 05^{\prime}$ 、东经 $122^{\circ} 12^{\prime} \sim 126^{\circ} 20^{\prime}$, 三 面群山环抱, 西部是大兴安岭, 北部是伊勒呼 里山和小兴安岭, 东部是长白山系的张广才岭, 南面横卧着的低丘为松辽分水岭, 是松花江、 嫩江、洮儿河和霍林河冲积作用为主而形成的 平原, 是我国三大平原之一东北平原的主要组 成部分。研究区处于西风带内, 天气变化比较 频繁, 年降水量为 $350 \sim 500 \mathrm{~mm}$, 干燥度 1. 1 1.5 , 为草甸草原带。松嫩平原由于土壤类型 的多样, 因此, 植被类型也多样, 是欧亚草原 带植被类型最丰富的地带。主要的优势种有羊 草、芦苇、小花碱茅、罗布麻、全叶马兰和寸 草苔等。本文数据主要来自于松嫩草原内分布 的 15 个气象站点 (图 1) 的从 1960.1.12014.12.1 的日气温和日降水量数据。

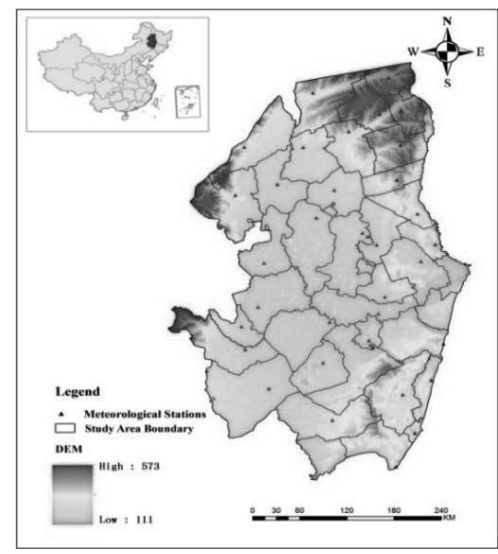

图 1 研究区示意图和气象站点分布图 


\section{3. 研究方法}

3.1 标准化蒸散指数 ( SPEI)

Vicente-serrano[1]等人构建了 SPEI 指数, 该指数同时具备了 SPI 和帕尔默干旱指数 (PDSI) 的优点。并且该指数具有 SPI 的多时 间尺度计算的优点, 可以识别不同历时的干早。 由于 SPEI 通过概率密度函数求解累积概率, 再将累积概率标准正态化而得, 消除了降水和 气温的时空分布差异, 在不同区域和时段均能 有效地反映干旱状况。详细信息可以参考 [1], 因篇幅有限不再细述。

\section{2 基于游程理论的干旱识别}

游程理论也叫轮次理论, 是分析时间序列的一 种最有效的方法。游程理论是指在连续出现的 同类事件过程中, 它的前后发生的是另外一类 事件, 如旱涝、连续无雨日、有雨日、河流水 丰枯交替出现等自然现象。本论文对干旱事件 的识别利用游程理论方法。SPEI 作为识别干 旱指标, 对照干旱本身的特点, 便可以把一次 负游程认为一次干旱事件, 一个干旱事件从开 始到结束的时间定义为干旱历时, 而负游程的 值为本次干旱事件的强度。计算公式为:

$$
S=-\sum_{i=1}^{\mathrm{d}} S P E I_{i}
$$

在干旱过程识别中, 通过程序识别出在植被生 育期 4-9 月这期间的干旱事件。

\section{3 Copula 函数}

Copula 作为一种全新的、适用性极强的相关 性度量工具, 是由 Sk1ar [2] 在 1959 第一次提 出。Sklar 定理是 Copula 函数研究领域中最 重要的一个定理, 它证明了 Copula 函数存在 的唯一性。即假设 $X$ 和 $Y$ 是两个随机变量, 它 们的联合概率分布函数为 $G(x, y)$, 如果它们 的边缘分布函数分别为 $F_{x}(x)$ 和 $F_{y}(y)$, 则一定 存在着连接函数 Copula $\mathrm{C}$, 满足 $\mathrm{G}(\mathrm{x}, \mathrm{y})=$ $\mathrm{C}\left(F_{x}(x), F_{y}(y)\right)$ 。如果 $F_{x}(x)$ 和 $F_{y}(y)$ 是连续的, 那么 $\mathrm{C}$ 是唯一的. 相反, 如果 C 是一个 Copula, $F_{x}(x)$ 和 $F_{y}(y)$ 是分布函数, 则由 $\mathrm{G}(\mathrm{x}, \mathrm{y})=$ $\mathrm{C}\left(F_{x}(x), F_{y}(y)\right)$ 所定义的 $\mathrm{G}(\mathrm{x}, \mathrm{y})$ 是一个联合 概率分布函数, 并且其边缘分布函数分别是 $F_{x}(x)$ 和 $F_{y}(y)$ 。

Copula 函数种类多样, 各有特点, 详细
信息可以参考 [3、4、5], 本文选用 Frank、 $\mathrm{G}-\mathrm{H} 、 \mathrm{Calyton}$ 等三种 Copula 函数联接干旱历 时和干旱严重程度的边缘分布函数, 并利用 RMSE [6] 和 AIC [7]进行优度检验。

\section{4 重现期}

重现期代表了某次干旱强度和干旱历时的干 旱风险连续出现两次的平均时间间隔，是某 一干旱历时和干旱强度下的干旱风险发生概 率的倒数。因此干旱历时和干旱强度分别超 过某一数值的重现期, 计算公式如下:

$$
\begin{gathered}
T(t)=\frac{E(L)}{P(T\rangle t)}=\frac{E(L)}{1-F_{T}(t)} \\
T(d)=\frac{E(L)}{P(D\rangle d)}=\frac{E(L)}{1-F_{D}(d)}
\end{gathered}
$$

考虑干旱历时和干旱强度联合分布的干旱风 险重现期有两种情形: $T>t$ 或和 $\mathrm{T}>\mathrm{d}$ 且 $D>d$,

分别记为 $\left.T_{\cup}(t, d), T_{\cap}(t, d) D\right\rangle d$ 。 SHIAU （2003）[8]推导出计算公式如下:

$$
\begin{aligned}
& T_{\cup}(t, d) \\
& =\frac{E(L)}{P(T\rangle t \cup D\rangle d)}=\frac{E(L)}{1-F_{T, D}(t, d)} \\
& =\frac{E(L)}{1-C\left(F_{T}(t), F_{D}(d)\right)} \\
& T_{\cap}(t, d)=\frac{E(L)}{P(T\rangle t \cap D\rangle d)}= \\
& \frac{E(L)}{1-F_{T}(t)-F_{D}(d)+C\left(F_{T}(t), F_{D}(d)\right)}
\end{aligned}
$$

本文以月为计算单位, 因此重现期也应以月为 单位。由上述一系列公式可以估计出某一时间 段的干旱历时、干旱强度的重现水平。

\section{4. 结果与分析}

4. 1. 基于游程理论的干旱识别

与 SPI 一样, SPEI 是基于概率计算得到的, 因此 SPI 的干旱等级划分标准 SPEI 同样适 用, 根据中华人民共和国 《气象干旱等级》 [9]国家标准来划分干旱等级, 如表 1 所示。 SPEI 可根据不同尺度进行计算, 一般有 $1 、 3 、$ 
Risk Analysis and Crisis Response in Big Data Era (RAC-16)

6、12、24 和 48 个月时间尺度。由于松嫩草 地一年中降水主要集中在生长季 的( 6-8 月), 生长季又是温度较高的夏半年, 蒸发旺盛, 因
此为较好的识别干旱情况, 本文选用 3 个月时 间尺度的 SPEI-3。

表 1 干旱等级划分

\begin{tabular}{lll}
\hline 等级 & 类型 & SPEI 值 \\
\hline 1 & 无旱 & $-0.5<\mathrm{SPEI}$ \\
2 & 轻早 & $-1.0<\mathrm{SPEI} \leqslant-0.5$ \\
3 & 中早 & $-1.5<\mathrm{SPEI} \leqslant-1.0$ \\
4 & 重旱 & $-2.0<\mathrm{SPEI} \leqslant-1.5$ \\
5 & 特早 & SPEI $\leqslant-2.0$ \\
\hline
\end{tabular}

表2 基于游程理论的干早识别结果

\begin{tabular}{lllllllll}
\hline 站名 & 站号 & 经度 & 纬度 & 海拔 & $\begin{array}{l}\text { 干旱事件 } \\
\text { 次数 }\end{array}$ & 平均历时 & 平均强度 & 最大强度 \\
\hline 乾安 & 50948 & 124.02 & 45.00 & 146.30 & 157 & 2.36 & 1.67 & 6.85 \\
克山 & 50658 & 125.88 & 48.05 & 234.60 & 173 & 1.99 & 1.53 & 4.49 \\
前郭 & 50949 & 124.87 & 45.08 & 136.20 & 159 & 2.04 & 1.68 & 6.43 \\
北安 & 50656 & 126.52 & 48.28 & 269.70 & 157 & 2.14 & 1.64 & 14.34 \\
双辽 & 54142 & 123.53 & 43.50 & 114.90 & 174 & 1.95 & 1.53 & 9.32 \\
安达 & 50854 & 125.32 & 46.38 & 149.30 & 165 & 2.06 & 1.66 & 7.10 \\
扶余 & 54063 & 126.00 & 44.97 & 196.80 & 173 & 1.92 & 1.55 & 8.33 \\
富裕 & 50742 & 124.48 & 47.80 & 162.70 & 169 & 1.95 & 1.58 & 6.06 \\
明水 & 50758 & 125.90 & 47.17 & 247.20 & 160 & 2.14 & 1.64 & 8.93 \\
泰来 & 50844 & 123.42 & 46.40 & 149.50 & 170 & 1.96 & 1.60 & 7.89 \\
白城 & 50936 & 122.83 & 45.63 & 155.30 & 175 & 1.93 & 1.52 & 7.71 \\
通榆 & 54041 & 123.07 & 44.78 & 149.50 & 165 & 1.99 & 1.59 & 6.85 \\
长岭 & 54049 & 123.97 & 44.25 & 188.90 & 174 & 1.96 & 1.50 & 7.72 \\
长春 & 54161 & 125.22 & 43.90 & 236.80 & 165 & 1.98 & 1.60 & 9.18 \\
齐齐哈尔 & 50745 & 123.92 & 47.38 & 147.10 & 165 & 1.99 & 1.62 & 8.85 \\
\hline
\end{tabular}

由表 2 可知, 各个气象站点间的干早事件次

数、平均历时、平均强度和最大强度差距不 是很明显, 说明各站点的干旱平均水平大体 相当。54 年间白城站点发生的干旱事件是最 多的, 然而乾安站点和北安站点的干旱事件 是相对较少的, 但是乾安站点的平均干旱历 时最长, 白城站点的平均干旱历时最短, 这 个现象正好跟干旱事件次数是相反规律。最 大强度依次发生在北安站点、双辽站点和长 春站点。可见, 各站的干旱特性具有显著 差异性, 研究它们之间的关系对草原干旱空 间特征分析具有重要意义。
4.2 Copula 函数分析

依据上述方法可以得到各个气象站点干早 历时和干旱强度的历史样本 (1960-2014 年), 对于干旱历时和干旱强度分布类型每个研 究学者有不同的见解, 比如说 Zenlenhastic 和 Salvai[10]以及 Shiau 和 Hsieh[11]指 出干旱历时符合指数分布, 而干旱强度则符 合 verGamma 分布。本研究中, 通过 $K-S$ 方 法 [12] 来拟合两个特征变量干旱历时和干 旱强度的边缘分布函数。结果发现干旱历时 符合 Gamma 分布函数, 干旱强度符合广义极 值分布 (GEV) 函数。Zeng X Ket al., [13] 等比较几种拟合方法发现众多拟合方法中 $\mathrm{K}-\mathrm{S}$ 方法具有其独特的优势, 因此其拟合结 
果具有可靠性。

由上述方法得到的干旱历时和干旱强度的 边缘分布, 但是因二者的分布函数类型不一 致, 可以通过 Copula 函数得打二者的联合 分布概率。因 Copula 函数的有多种类型, 选择不同类型的 Copula 函数计算得到的联 合分布存在明显的差异。因此, 本研究选取 比较常用的阿基米德 Copula 函数, 分别是 Frank 类型、 $\mathrm{G}-\mathrm{H}$ 类型和 Clayton 类型, 并 利用赤池信息量 准 则
(Akaike Information Criterion ( AIC )) 和均方根误差 (RMSE) 方法选择最优的 Copula 函数。结果发现, Frank 函数的 paramater、AIC、RMSE 系数最小, 因此选择 Frank Copula 函数构建松嫩草原干旱特征 变量的联合概率分布。因气象站点较多, 本 文中未能一一列出各站点的分布情况, 图 2 为随机选取长岭气象站点的联合同现期三 维图、联合重现期的三维图和联合重现期的 等值线图。


图 2 长岭气象站点的联合同现期三维图、联合重现期三维图和联合重现期等值线图（从左 到右）

4.3. 重现期空间分布特征分析

图 3 和图 4 为指定重现期下的各站点干旱 历时和干旱强度的联合重现期和同现重现 期的空间分布特征。从图 3 可知, 重现期为 10 个月、20 个月和 50 个月下的联合重现期 空间分布特征变化基本一致, 重现期为 5 个 月的联合重现期稍微有差别, 但是最大差别 不是很明显。从图 4 可知, 重现期为 10 个 月、20个月和 50 个月的同现重现期有一致 的空间分布特征变化, 然而重现期为 5 个月 的同现重现期的空间分布特征有明显的差
别, 但是最大差别还是不是很明显。在不同 重现期下, 北安、明水和乾安对应的联合重 现期大于其他站点对应的联合重现期, 然而 在安达、扶余和前郭对应的联合重现期是最 小的。在不同重现期条件下, 各站点对应的 干旱历时和干旱强度的同现重现期变化与 对应联合重现期空间变化基本相反, 具体情 况是白城、扶余、肇东和富裕对应的同现重 现期最大, 北安和乾安对应的同现重现期最 小。可见, 干旱空间特征分析能够为区域干 旱灾害风险管理提供有效的科学依据。

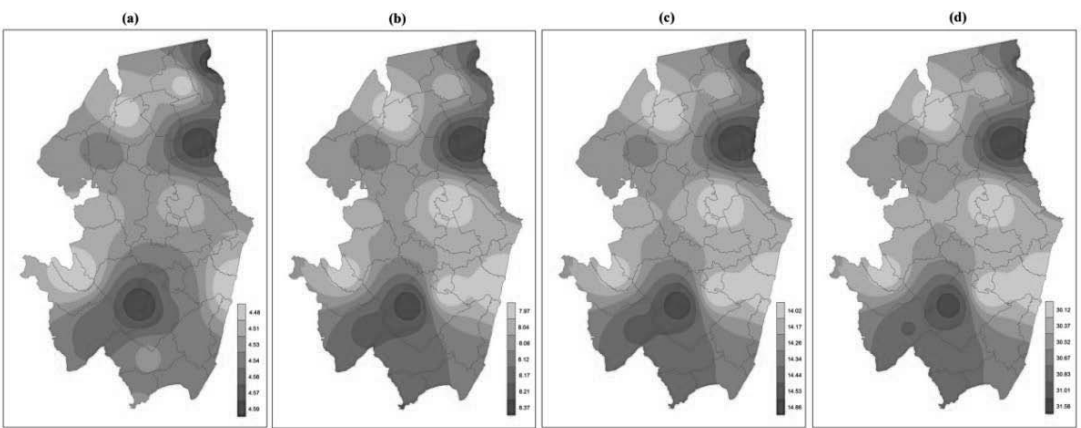

图 3a、b、c、d 分别是松嫩草原干旱在指定重现期 5 个月、10个月、20 个月、50个月下的 联合重现期的空间分布特征 
Risk Analysis and Crisis Response in Big Data Era (RAC-16)

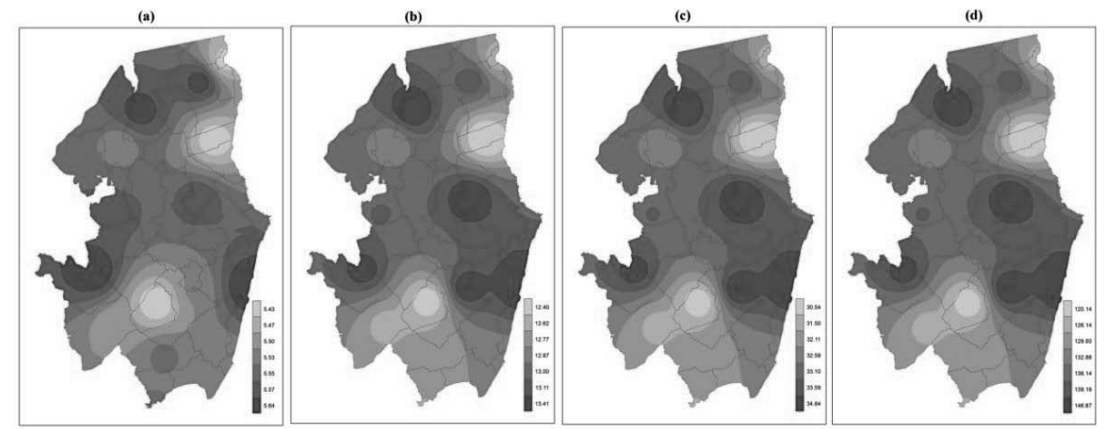

图 $4 a 、 b 、 c 、 d$ 分别是松嫩草原干旱在指定重现期 5 个月、10 个月、20 个月、50个月下 的同现重现期期的空间分布特征

\section{5. 结论}

本文以中国北方草原松嫩草地为研究区, 选取干旱历时和干旱强度为干旱特征变量 并利用游程理论对其进行识别，根据 Copula 函数建立了两者之间的联合分布 函数并计算其重现期, 最后探讨了指定重 现期下的干旱重现期和同现期的空间分布 情况。研究表明: Copula 函数为研究多变 量干旱特征分析提供了有效的手段, 弥补 了传统干旱单变量特征分析的不足。通过 优度拟合分析发现 Frank copula 函数对各 站干旱特征变量间的联合分布具有较好的 拟合结果。通过分析重现期空间分布特征 发现, 各站点具有显著的空间分布特性, 各站点干旱重现期的差异较大。通过对重 现期的深入研究能够为松嫩草地干旱灾害 风险评价与管理工作提供科学依据。

\section{Acknowledgements}

This study is supported by the National Key Technology R\&D Program of China under Grant Nos.2013BAK05B02 and 2013BAK05B01 and the National Non-Profit Research Program of China (No.201401015).

\section{致谢}

本研究得到了 “十二五”国家科技支撑项目 （2013BAK05B02、2013BAK05B01）和水 利部公益性行业科研专项经费项目 （201401015）的资助。

\section{参考文献}

[1] S.M. Vicente-Serrano, S. Beguería, J.I. López-Moreno, A multi-scalar drought index sensitive to global warming: The Standardized Precipitation Evapotranspiration Index-SPEI, Journal of Climate. 23(7): 1696-1718, 2010.

[2]A. Sklar. Fonctions de re 'partition a 'n dimensions et leursmarges, Publ. Inst. Stat University Paris, Paris France. pp 229-231, 1959.

[3] A. Sklar. Random variables, joint distribution functions, and copulas, Kybernetika -Praha.

9(6): 449-460, 1973.

[4] R. B. Nelsen. An introduction to copulas, Technometrics. 42(4): xx 315, 2000.

[5] G. Salvadori, C.D. Michele, N.T. Kottegoda, et al. Extremes in Nature: An Approach Using Copulas, Berlin Springer 49(1): 5-89, 2007.

[6] C.J. Willmott. On the validation of models, Physical Geography. 2:184-194, 1981.

[7] H. Akaike. A new look at the statistical model identification, Automatic Control IEEE Transactions on. 19(6): 716-723, 1974.

[8] J.T. Shiau. Return period of bivariate distributed extreme hydrological events, Stochastic Environmental Research \& Risk Assessment. 17(1):42-57, 2003. 
Risk Analysis and Crisis Response in Big Data Era (RAC-16)

[9]National Standard of People's Republic of China GB/T 20481-2006 the grade of meteorological drought Beijing China Standard Press. (2006) (in Chinese).

[10]Zelenhasić E, Salvai A. A method of streamflow drought analysis. Water Resour Res 23(1) (1987)156-168.

[11]Shiau J, Shen H. Recurrence analysis of hydrologic droughts of differing severity. J Water Resour Plan Manag 127(1):30-40,
2001.

[12] J. Frank, J. Massey. The KolmogorovSmirnov Test for Goodness of Fit, Journal of the American Statistical Association. 46(253):68-78, 2012.

[13]X .K. Zeng, D. Wang, J.C. Wu. Evaluating the Three Methods of Goodness of Fit Test for Frequency Analysis. Journal of Risk Analysis and Crisis Response. 5(3) (2015); 178-187. 